\title{
Monocular Energy Spectrum using the TAx4 Fluorescence
} Detector

\section{Mathew Potts* and Charles Jui on behalf of the Telescope Array Collaboration} (a complete list of authors can be found at the end of the proceedings)

University of Utah,

201 Presidents' Cir, Salt Lake City, USA

E-mail: matt.potts@cosmic.utah.edu, cchjui@gmail.com

Following the evidence for a hotspot in the arrival directions of the highest energy cosmic rays, the Telescope Array (TA) Experiment undertook the TAx4 upgrade to expand the area of our Surface Detectors (SD) by a factor of 4 and have added new Fluorescence Detector (FD) stations to view over the new SD arrays. Currently, TAx 4 consists of 12 FDs and 257 SDs, of a planned 500, at a spacing of $2.08 \mathrm{~km}$ spread over two sites. TAx4 North (4 FDs), completed in 2018, views over the northern wing of the new SD, and TAx4 South (8 FDs), completed in 2019, views over the southern wing. Both FD sites are in routine observation, with data being taken remotely at the TAx4 South site. In this work, we will report on the performance of the TAx4 FD, showing data/MC comparisons. We will present a preliminary monocular energy spectrum for the TAx4 fluorescence detector and the progress of the hybrid analysis.

$37^{\text {th }}$ International Cosmic Ray Conference (ICRC 2021)

July 12 th $-23 r d, 2021$

Online - Berlin, Germany

\footnotetext{
*Presenter
} 


\section{Introduction}

The Telescope Array (TA) experiment, located in Millard County, UT, USA (Figure 1), is the largest cosmic ray detector in the Northern hemisphere designed to observe ultra-high energy cosmic rays (UHECRs). The experiment consist of 867 surface detectors (SDs) and 56 fluorescence detector (FD) telescopes. All SDs used in the experiment are composed of 2 layers of $3 \mathrm{~m}^{2} \times 1.2 \mathrm{~cm}$ plastic scintillator [3]; however, the SD spacing and electronics varies depending on the array the SD is part of. The FDs, with 256 photo-multiplier tubes (PMTs) each, are spread across 3 arrays: the main TA array, TA Low Energy extension (TALE) array, and the recently added TA times four (TAx4) array following evidence of a hotspot in the arrival directions of UHECRs [1, 2].

The TAx4 array contains 12 FDs and 257 SDs, of a planned 500, at a spacing of $2.08 \mathrm{~km}$ spread over two sites. The spacing of the SDs was chosen to optimize our detection of events at the highest energy range, $E>20 \mathrm{EeV}$. There are 4 FDs looking over the northern group of SDs, called TAx 4 North. These FDs view a range of $4^{\circ}-17^{\circ}$ in elevation and $12^{\circ}-76^{\circ}$ in azimuth (North of East). TAx4 North was completed in early-2018 and has been taking data regularly since June, 2018. TAx4 South views over the southern group of SDs. TAx4 South views the same elevation range as TAx4 North and covers a range of $238^{\circ}-350^{\circ}$ in azimuth (North of East). Construction on

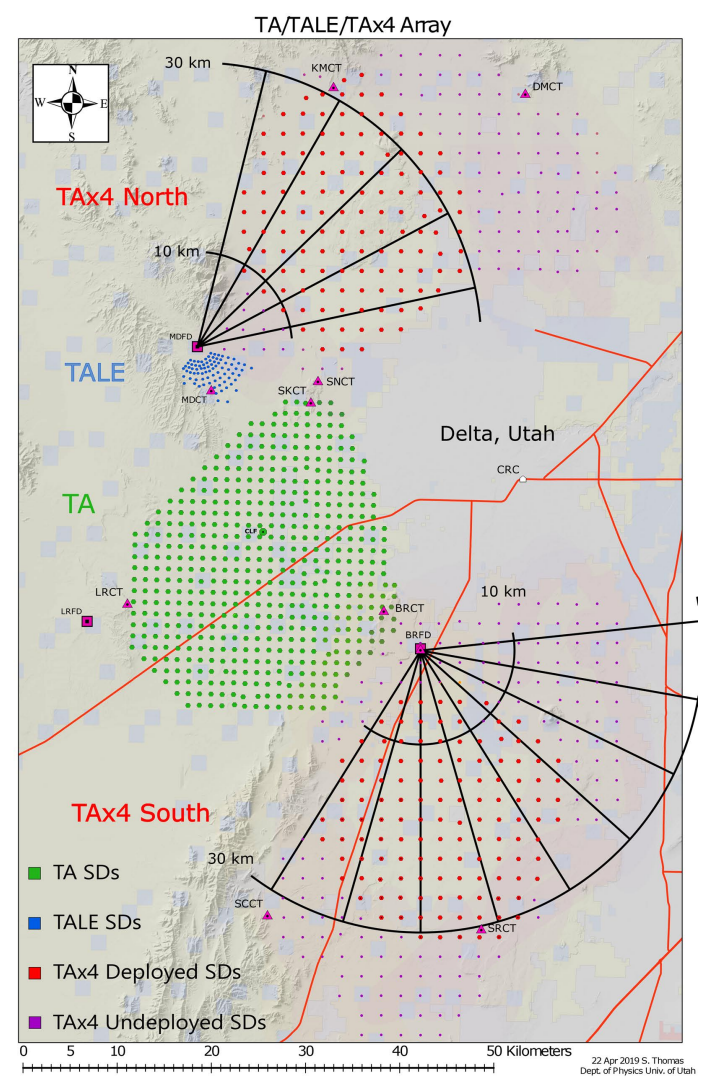

Figure 1: Map of the entire TA experiment. The magenta triangles are locations of communication towers and the magenta squares are FD sites. The 257 deployed TAx4 SDs are shown as red circle to the northeast and southeast. While the purple circles are planned location of the remaining 243 TAx4 SDs to be deployed. The 2 fan shapes drawn with black lines show the field of view of TAx4 FDs. 
this site was competed in mid-2019 and has been taking regular data since October, 2019.

Since the completion of TAx 4 there has been two upgrades to the FDs, a vertical shower trigger enhancement in April, 2019 and inter-mirror trigger in June, 2019. This work uses data after both upgrades were completed. We will report on the performance of the TAx4 FDs, showing resolutions and data-MC comparisons and present a preliminary monocular energy spectrum using the TAx4 FDs. We will also discuss the progress towards a hybrid energy spectrum.

\section{Monocular Reconstruction and Quality Cuts}

The data set used in this work is from both TAx4 sites from June 26, 2019 to April 19, 2021, approximately 2 years of data. TAx4 North has a total detector on-time is 1184.1 hours, with 696.9 hours of it being good weather on-time. Where good weather on-time is defined by no clouds being overhead and no horizon clouds in the field of view of each site. TAx4 South has a total detector on-time of 613.6 hours, with 354.9 hours being good weather on-time.

The events that are reconstructed in monocular mode have their geometry determined by the equation,

$$
t_{i}=t_{0}+\frac{R_{p}}{c} \cdot \tan \left(\frac{\pi-\psi-\chi_{i}}{2}\right),
$$

where $t_{i}$ and $\chi_{i}$ are the trigger time and pointing direction of tube $i$, respectively; $\psi$ is the in-plane angle; $\mathrm{R}_{p}$ is the impact parameter of the shower; $c$ is the speed of light; and $t_{0}$ is the time when the shower is calculated to be at $\mathrm{R}_{p}$.

The profile of the extensive air shower is fitted using the Gaisser-Hillas parameterization formula [4],

$$
N(x)=N_{\max } \cdot\left(\frac{x-X_{0}}{X_{\max }-X_{0}}\right)^{\frac{X_{\max -X_{0}}}{\lambda}} \cdot e^{\frac{X_{\max -x}}{\lambda}}
$$

where $N(x)$ is the number of charged particles at a given slant depth, $x$, in $\mathrm{g} / \mathrm{cm}^{2} ; X_{\max }$ is the slant depth where the number of the secondary particles reaches the maximum; $N_{\max }$ is the maximum number of particles at $X_{\max } ; X_{0}$ is a fit parameter associated with the depth of the first interaction; and $\lambda$ is $70 \mathrm{~g} / \mathrm{cm}^{2}$. Events with lower energies may have too short of track lengths in order to carry out geometry reconstruction alone. For these cases, a profile-constrained geometrical fit is applied to the reconstructed events.

\begin{tabular}{cc}
\multicolumn{2}{c}{ Event Reconstruction Cuts } \\
\hline \hline Rayleigh Filter & $\mathrm{P}_{\log _{10}} \geq 2$ \\
Brightness Cut & $\Sigma N_{\gamma} / N_{\text {ngtube }} \geq 200$ \\
Track length & $>7.9^{\circ}$ \\
Track width RMS & $\theta_{\mathrm{RMS}} \leq 1^{\circ}$ \\
Profile Fit & $\chi^{2} / \mathrm{ndf}<14$ \\
Angular Speed Cut & $\leq 5.73 \mu \mathrm{s} / \mathrm{deg}$ \\
First Interaction & $\mathrm{X}_{0} \leq 1200 \mathrm{~g} / \mathrm{cm}^{2}$ \\
\hline \hline
\end{tabular}

Table 1: TAx4 monocular event reconstruction quality cuts. 
In order to obtain good resolutions and data-Monte Carlo comparisons, quality cuts are applied to the fully reconstructed data set. The quality cuts used in this work can be seen on Table 1 .

\section{Monte Carlo Simulations}

A CORSIKA (COsmic Ray SImulations for KAscade) shower library with QGSJetII-03 protons is used to produce the Monte Carlo (MC) set. The shower energies are thrown between $10^{17}-10^{21} \mathrm{eV}$ with a spectral index of 2. The lower spectral index was chosen to increase the MC statistics at the highest energies. Events were generated uniformly in a $50 \mathrm{~km}$ circle around each detector site down to a zenith angle of $70^{\circ}$. For each data part, $5 \times 10^{4} \mathrm{MC}$ events were thrown; however, the exact number thrown for a given data part was adjusted based on the on-time of the data part being simulated. The MC used atmospheric profiles implemented from the GDAS database which provides a value of the atmospheric vertical aerosol optical depth in 3 hour increments.

The resolutions for both TAx 4 sites were found to be similar, so this work will only show TAx4 North's resolutions. Figure 2, shows TAx4 North's resolution histograms for energy, zenith angle, psi, and impact parameter. For events with energy greater than $10^{18.5} \mathrm{eV}$, we obtain resolutions of $\sim 20 \%$ in energy, $\sim 3^{\circ}$ in zenith angle, $\sim 7^{\circ}$ in $\psi$ angle, and $\sim 11 \%$ in the impact parameter.
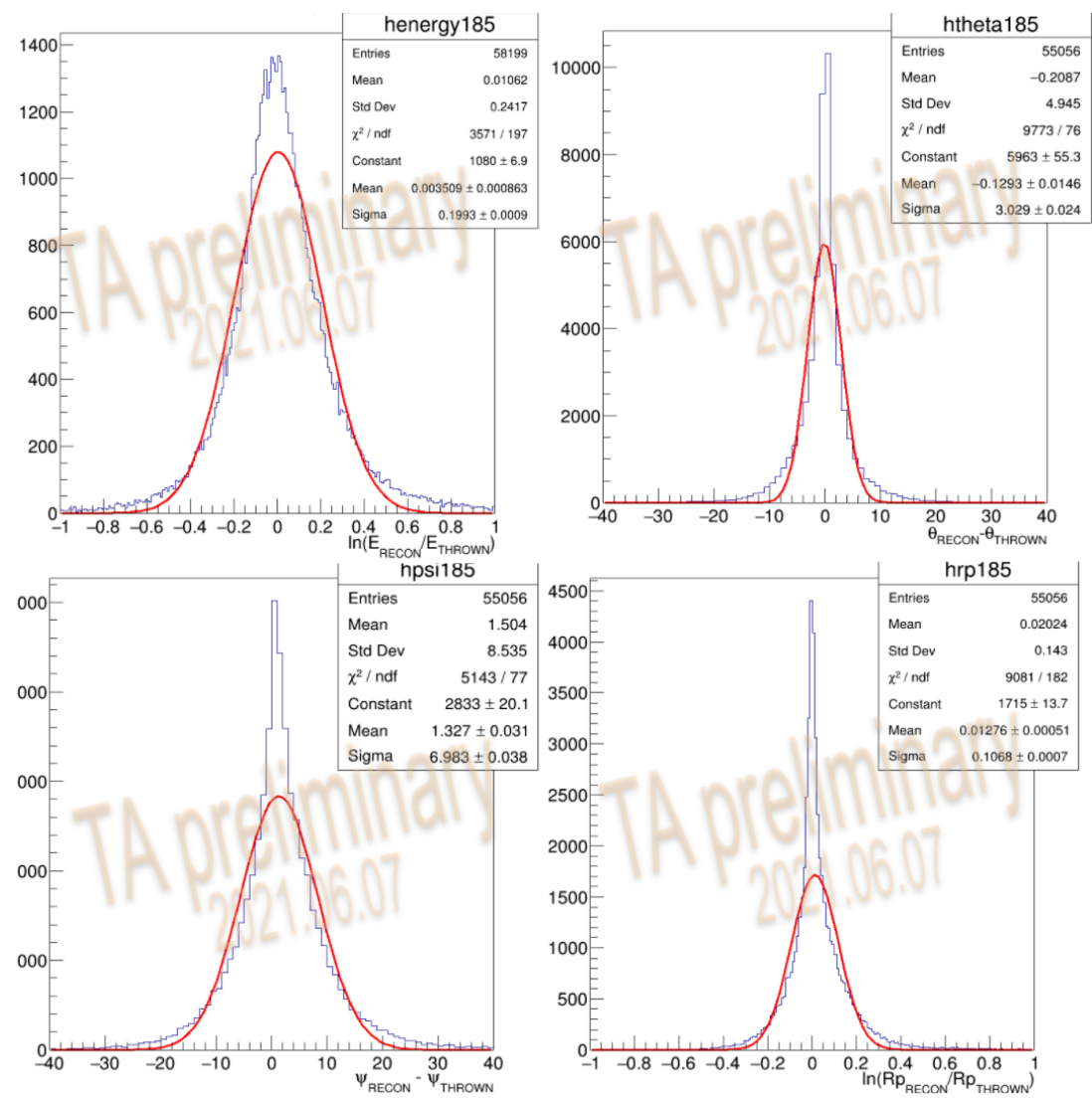

Figure 2: Resolutions Histograms for TAx4 North. Top Left: Energy resolution. Top Right: Zenith Angle, $\theta$, resolution plot. Bottom Left: In-plane Angle, $\psi$, resolution. Bottom Right: Impact parameter, $R_{p}$, resolution. 

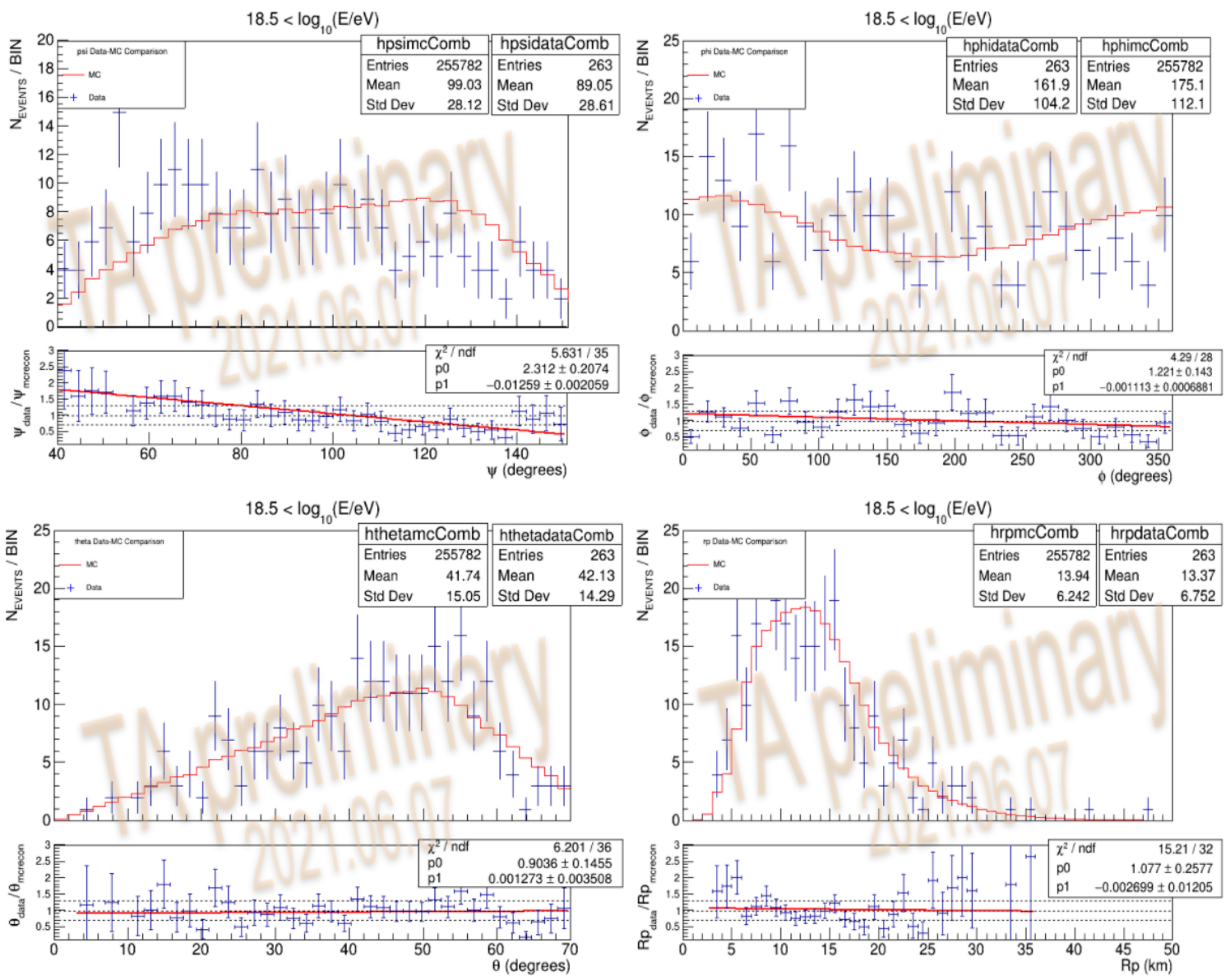

Figure 3: TAx4 Data-MC comparisons. Top Left: in-plane angle, $\psi$. Top Right: the azimuthal angle, $\phi$. Bottom Left: the zenith angle, $\theta$. Bottom Right: the impact parameter, $R_{p}$. The blue points with error bars show the data, while the MC is shown by the red histogram. The Monte Carlo has been normalized to the same number of events as the data. Below each histogram are ratio plots of the data divided by the MC fitted with a line to determine agreement.

\section{Data-MC Comparisons}

To check if the MC reasonably represents the data, measurable parameters' are plotted and compared. The impact parameter and zenith angle parameters are particularly important to determining the detector's aperture. Figure 3 data-mc comparisons for the in-plane angle $(\psi)$, impact parameter $\left(R_{p}\right)$, azimuthal angle $(\phi)$, and zenith angle $(\theta)$. Below each histogram are ratio plots of the data divided by the $\mathrm{MC}$, with a fitted line to determine agreement. All the geometrical parameters appear to be in reasonable agreement with the MC.

\section{TAx4 Monocular Spectrum}

Calculating the TAx4 monocular energy spectrum is straight forward, due to there being no overlap of the field of views of each site. The total number of events in each energy bin is given by,

$$
N_{\mathrm{TAx} 4}\left(E_{i}\right)=N_{N}\left(E_{i}\right)+N_{S}\left(E_{i}\right)
$$




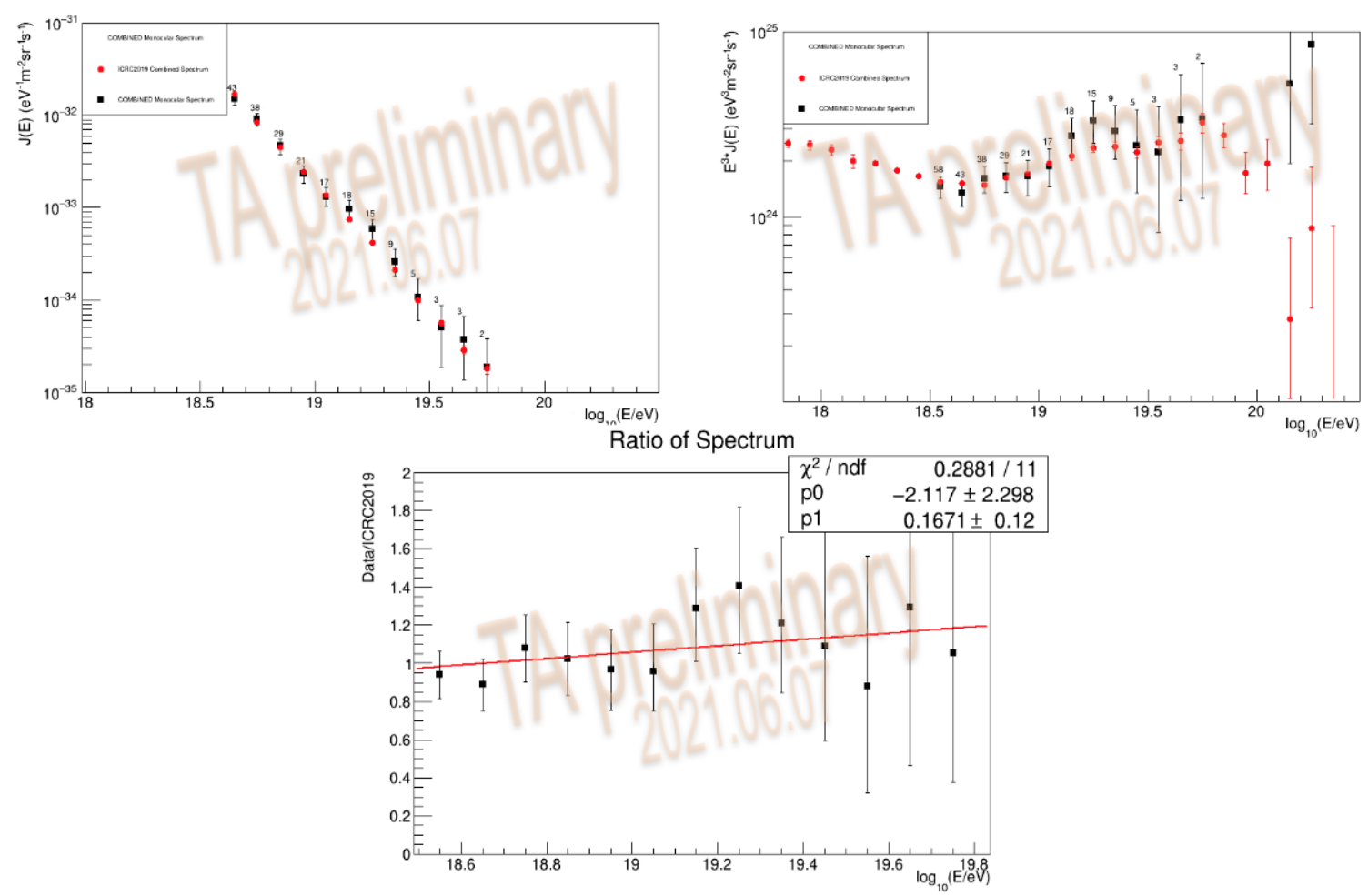

Figure 4: TAx4 Monocular Spectrum. The top figures show the ICRC2019 TA combined spectrum, the red points, being compared to TAx4 monocular spectrum, the black points. Above each TAx4 data point the number of cosmic ray events in that energy bin is printed. Top Left: Cosmic ray flux, $J(E)$. Top Right: Flux multiplied by $\mathrm{E}^{3}$ to bring out spectral features. Bottom: Ratio of the TAx4 and ICRC2019 TA combined spectra.

where $N_{N}\left(E_{i}\right)$ and $N_{S}\left(E_{i}\right)$ are the number of events for TAx4 North and TAx4 South respectively.

The total exposure of the TAx 4 detector, $\epsilon_{\mathrm{TAx} 4}$, also simplifies to a summation of each sites exposure,

$$
\epsilon_{\mathrm{TAx} 4}=A \Omega_{N} T_{N}+A \Omega_{S} T_{S}
$$

where $\mathrm{A} \Omega_{N}$ and $\mathrm{A} \Omega_{S}$ are the apertures for TAx4 North and TAx4 South respectively; $T_{N}$ and $T_{S}$ are the good weather on-time for TAx4 North and TAx4 South repectively.

The TAx 4 cosmic ray flux, $J_{\mathrm{TAx} 4}\left(E_{i}\right)$, is given by,

$$
J_{\mathrm{TAx} 4}\left(E_{i}\right)=\frac{N_{\mathrm{TAx} 4}\left(E_{i}\right)}{\Delta E_{i} \cdot \epsilon_{\mathrm{TAx} 4}\left(E_{i}\right)},
$$

where $\Delta E_{i}$ is the width of the energy bin. The flux of cosmic rays follows a power law distribution with a spectral index of $\sim 3$; therefore, the spectral features of the energy spectrum can be brought out by multiplying $J_{\mathrm{TAx} 4}$ by $E^{3}$. Figure 4 shows this works monocular energy spectrum compared with the ICRC2019 TA combined spectrum. The bottom plot in Figure 4 is a ratio of both spectra that shows agreement with this work's monocular spectrum and the ICRC2019 TA combined spectrum at and above $10^{18.65} \mathrm{eV}$. 


\section{Summary and Plans}

The TAx4 North and TAx4 South sites were completed in 2018 and 2019, respectively. Both sites are now taking data continuously on clear moonless nights. For this work, we used approximately 2 years of data from June, 2019 to April, 2021 to generate a monocular energy spectrum using the TAx4 detector. The cosmic ray energy spectrum measured by TAx 4 is in agreement with the ICRC2019 TA combined spectrum. The data-MC comparisons for energy and geometrical variables are in reasonable agreement.

A monocular energy spectrum is the first step towards my goal of generating a hybrid energy spectrum using the TAx4 detector. Hybrid reconstruction takes into account the SD timing information as well tube timing and uses it to constrain an event's geometry. We have used hybrid reconstruction on the data (Figure 5), but we are still waiting for full hybrid Monte Carlo before proceeding with a hybrid analysis. Currently, we are using a parametric hybrid MC program to estimate the detector's hybrid aperture and resolutions, while implementation of a full hybrid MC is in progress.
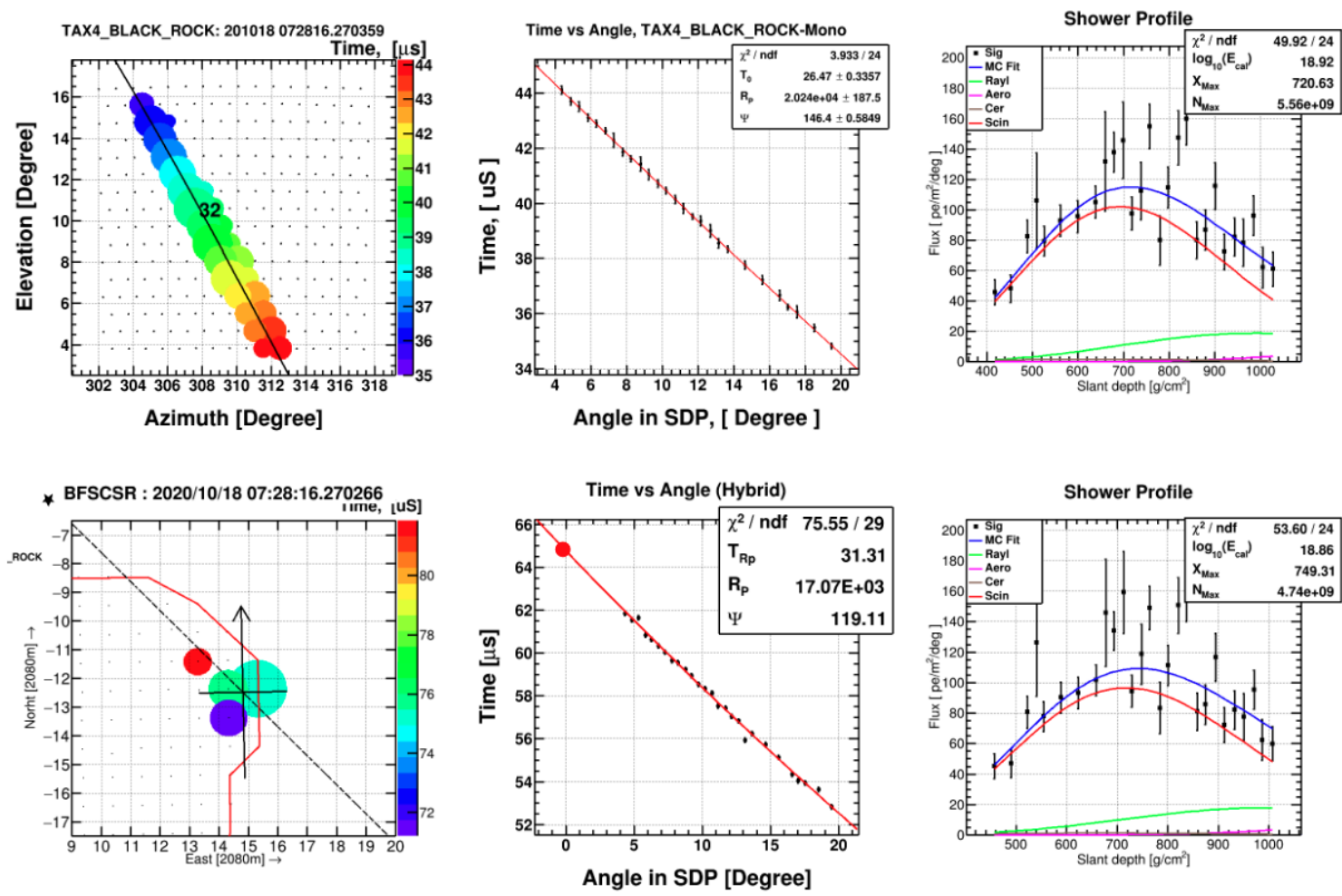

Figure 5: The same TAx4 South event reconstructed using monocular (top row) and hybrid reconstruction (bottom row). Top Left: FD display of the event. The size of the colored circles corresponds to the size of the signal, and color represents tube triggering time. Top Middle: Time verse angle plot using monocular reconstruction. Top Right: Shower profile using monocular reconstruction. Bottom Left: SD display of event. Bottom Middle: Time verse angle plot using hybrid reconstruction. The red data point is taken from the timing of the closest SD to the shower core. Bottom Right: Shower profile using hybrid reconstruction. 


\section{References}

[1] K. Kawata et. al. Updated Results on the UHECR Hotspot Observed by the Telescope Array Experiment, PoS(ICRC2019)310.

[2] R. Abbasi et al., Evidence of Intermediate-scale Energy Spectrum Anisotropy of Cosmic Rays $E \geq 10^{19.2} \mathrm{eV}$ with the Telescope Array Surface Detector, ApJ. 862, 91.

[3] T. Abu-Zayyad et al., The surface detector array of the Telescope Array experiment, NIM-A 689, 87A [astro-ph/1201.4964].

[4] T. K. Gaisser and A. M. Hillas, "Reliability of the method of constant intensity cuts for reconstructing the average development of vertical showers", in Proceedings of the International Cosmic Ray Conference, vol. 8 (1977) 353-357 


\section{Full Authors List: Telescope Array Collaboration}

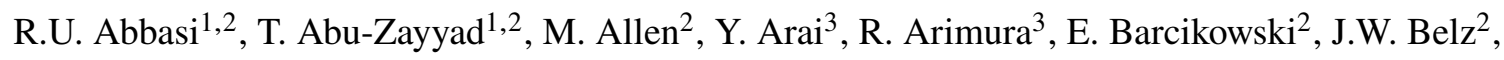
D.R. Bergman ${ }^{2}$, S.A. Blake², I. Buckland ${ }^{2}$, R. $\mathrm{Cady}^{2}$, B.G. Cheon ${ }^{4}$, J. Chiba ${ }^{5}$, M. Chikawa ${ }^{6}$, T. Fujii ${ }^{7}$, K. Fujisue ${ }^{6}$, K. Fujita ${ }^{3}$, R. Fujiwara ${ }^{3}$, M. Fukushima ${ }^{6}$, R. Fukushima ${ }^{3}$, G. Furlich ${ }^{2}$, R. Gonzalez ${ }^{2}$, W. Hanlon ${ }^{2}$, M. Hayashi ${ }^{8}$, N. Hayashida ${ }^{9}$, K. Hibino ${ }^{9}$, R. Higuchi ${ }^{6}$, K. Honda ${ }^{10}$, D. Ikeda ${ }^{9}$, T. Inadomi ${ }^{11}$, N. Inoue ${ }^{12}$, T. Ishii ${ }^{10}$, H. Ito ${ }^{13}$, D. Ivanov ${ }^{2}$, H. Iwakura ${ }^{11}$, A. Iwasaki ${ }^{3}$, H.M. Jeong ${ }^{14}$, S. Jeong ${ }^{14}$, C.C.H. Jui ${ }^{2}$, K. Kadota ${ }^{15}$, F. Kakimoto ${ }^{9}$, O. Kalashev ${ }^{16}$, K. Kasahara ${ }^{17}$, S. Kasami ${ }^{18}$, H. Kawai ${ }^{19}$, S. Kawakami ${ }^{3}$, S. Kawana ${ }^{12}$, K. Kawata ${ }^{6}$, I. Kharuk ${ }^{16}$, E. Kido ${ }^{13}$, H.B. Kim ${ }^{4}$, J.H. Kim ${ }^{2}$, J.H. Kim ${ }^{2}$, M.H. Kim ${ }^{14}$, S.W. Kim ${ }^{14}$, Y. Kimura ${ }^{3}$, S. Kishigami ${ }^{3}$, Y. Kubota ${ }^{11}$, S. Kurisu ${ }^{11}$, V. Kuzmin ${ }^{16^{*}}$, M. Kuznetsov ${ }^{16,20}$, Y.J. Kwon ${ }^{21}$, K.H. Lee ${ }^{14}$, B. Lubsandorzhiev ${ }^{16}$, J.P. Lundquist ${ }^{2,22}$, K. Machida ${ }^{10}$, H. Matsumiya ${ }^{3}$, T. Matsuyama ${ }^{3}$, J.N. Matthews ${ }^{2}$, R. Mayta ${ }^{3}$, M. Minamino ${ }^{3}$, K. Mukai ${ }^{10}$, I. Myers ${ }^{2}$, S. Nagataki ${ }^{13}$, K. Nakai ${ }^{3}$, R. Nakamura ${ }^{11}$, T. Nakamura ${ }^{23}$, T. Nakamura ${ }^{11}$, Y. Nakamura ${ }^{11}$, A. Nakazawa ${ }^{11}$, E. Nishio ${ }^{18}$, T. Nonaka ${ }^{6}, \mathrm{H}_{\text {. Oda }}{ }^{3}, \mathrm{~S}_{\text {. Ogio }}{ }^{3,24}$, M. Ohnishi ${ }^{6}$, H. Ohoka ${ }^{6}$, Y. Oku ${ }^{18}$, T. Okuda ${ }^{25}$, Y. Omura ${ }^{3}$, M. Ono ${ }^{13}$, R. Onogi ${ }^{3}$, A. Oshima ${ }^{3}$, S. Ozawa ${ }^{26}$, I.H. Park ${ }^{14}$, M. Potts ${ }^{2}$, M.S. Pshirkov ${ }^{16,27}$, J. Remington ${ }^{2}$, D.C. Rodriguez ${ }^{2}$, G.I. Rubtsov ${ }^{16}$, D. Ryu ${ }^{28}$, H. Sagawa ${ }^{6}$, R. Sahara ${ }^{3}$, Y. Saito ${ }^{11}$, N. Sakaki ${ }^{6}$, T. Sako ${ }^{6}$, N. Sakurai ${ }^{3}$, K. Sano ${ }^{11}$, K. Sato ${ }^{3}$, T. Seki ${ }^{11}$, K. Sekino ${ }^{6}$, P.D. Shah ${ }^{2}$, Y. Shibasaki ${ }^{11}$, F. Shibata ${ }^{10}$, N. Shibata ${ }^{18}$, T. Shibata ${ }^{6}$, H. Shimodaira ${ }^{6}$, B.K. Shin ${ }^{28}$, H.S. Shin ${ }^{6}$, D. Shinto ${ }^{18}$, J.D. Smith ${ }^{2}$, P. Sokolsky ${ }^{2}$, N. Sone ${ }^{11}$, B.T. Stokes ${ }^{2}$, T.A. Stroman ${ }^{2}$, Y. Takagi ${ }^{3}$, Y. Takahashi ${ }^{3}$, M. Takamura $^{5}$, M. Takeda ${ }^{6}$, R. Takeishi ${ }^{6}$,

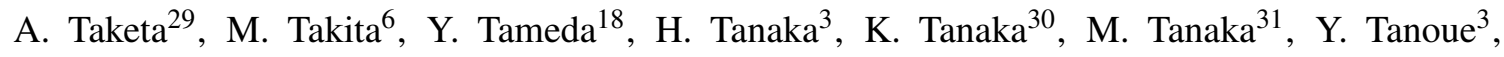
S.B. Thomas ${ }^{2}$, G.B. Thomson ${ }^{2}$, P. Tinyakov ${ }^{16,20}{ }^{\text {I. } \text { Tkachev }^{16} \text {, H. Tokuno }}{ }^{32}$, T. Tomida ${ }^{11}$, S. Troitsky ${ }^{16}$, R. Tsuda ${ }^{3}$, Y. Tsunesada ${ }^{3,24}$, Y. Uchihori ${ }^{33}$, S. Udo ${ }^{9}$, T. Uehama ${ }^{11}$, F. Urban ${ }^{34}$, T. Wong ${ }^{2}$, K. Yada ${ }^{6}$, M. Yamamoto ${ }^{11}$, K. Yamazaki ${ }^{9}$, J. Yang ${ }^{35}$, K. Yashiro ${ }^{5}$, F. Yoshida ${ }^{18}$, Y. Yoshioka ${ }^{11}$, Y. Zhezher ${ }^{6,16}$, and Z. Zundel ${ }^{2}$

${ }^{1}$ Department of Physics, Loyola University Chicago, Chicago, Illinois, USA

${ }^{2}$ High Energy Astrophysics Institute and Department of Physics and Astronomy, University of Utah, Salt Lake City, Utah, USA

${ }^{3}$ Graduate School of Science, Osaka City University, Osaka, Osaka, Japan

${ }^{4}$ Department of Physics and The Research Institute of Natural Science, Hanyang University, Seongdong-gu, Seoul, Korea

${ }^{5}$ Department of Physics, Tokyo University of Science, Noda, Chiba, Japan

${ }^{6}$ Institute for Cosmic Ray Research, University of Tokyo, Kashiwa, Chiba, Japan

7 The Hakubi Center for Advanced Research and Graduate School of Science, Kyoto University, KitashirakawaOiwakecho, Sakyo-ku, Kyoto, Japan

${ }^{8}$ Information Engineering Graduate School of Science and Technology, Shinshu University, Nagano, Nagano, Japan

${ }^{9}$ Faculty of Engineering, Kanagawa University, Yokohama, Kanagawa, Japan

${ }^{10}$ Interdisciplinary Graduate School of Medicine and Engineering, University of Yamanashi, Kofu, Yamanashi, Japan

${ }^{11}$ Academic Assembly School of Science and Technology Institute of Engineering, Shinshu University, Nagano, Nagano, Japan

12 The Graduate School of Science and Engineering, Saitama University, Saitama, Saitama, Japan

${ }^{13}$ Astrophysical Big Bang Laboratory, RIKEN, Wako, Saitama, Japan

${ }^{14}$ Department of Physics, SungKyunKwan University, Jang-an-gu, Suwon, Korea

15 Department of Physics, Tokyo City University, Setagaya-ku, Tokyo, Japan

${ }^{16}$ Institute for Nuclear Research of the Russian Academy of Sciences, Moscow, Russia

${ }^{17}$ Faculty of Systems Engineering and Science, Shibaura Institute of Technology, Minato-ku, Tokyo, Japan 
${ }^{18}$ Department of Engineering Science, Faculty of Engineering, Osaka Electro-Communication University, Neyagawashi, Osaka, Japan

${ }^{19}$ Department of Physics, Chiba University, Chiba, Chiba, Japan

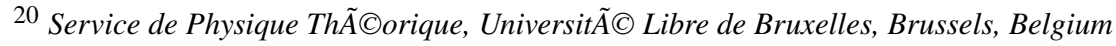

${ }^{21}$ Department of Physics, Yonsei University, Seodaemun-gu, Seoul, Korea

22 Center for Astrophysics and Cosmology, University of Nova Gorica, Nova Gorica, Slovenia

${ }^{23}$ Faculty of Science, Kochi University, Kochi, Kochi, Japan

${ }^{24}$ Nambu Yoichiro Institute of Theoretical and Experimental Physics, Osaka City University, Osaka, Osaka, Japan

${ }^{25}$ Department of Physical Sciences, Ritsumeikan University, Kusatsu, Shiga, Japan

${ }^{26}$ Quantum ICT Advanced Development Center, National Institute for Information and Communications Technology, Koganei, Tokyo, Japan

27 Sternberg Astronomical Institute, Moscow M.V. Lomonosov State University, Moscow, Russia

${ }^{28}$ Department of Physics, School of Natural Sciences, Ulsan National Institute of Science and Technology, UNIST-gil, Ulsan, Korea

${ }^{29}$ Earthquake Research Institute, University of Tokyo, Bunkyo-ku, Tokyo, Japan

${ }^{30}$ Graduate School of Information Sciences, Hiroshima City University, Hiroshima, Hiroshima, Japan

${ }^{31}$ Institute of Particle and Nuclear Studies, KEK, Tsukuba, Ibaraki, Japan

${ }^{32}$ Graduate School of Science and Engineering, Tokyo Institute of Technology, Meguro, Tokyo, Japan

${ }^{33}$ Department of Research Planning and Promotion, Quantum Medical Science Directorate, National Institutes for Quantum and Radiological Science and Technology, Chiba, Chiba, Japan

${ }^{34}$ CEICO, Institute of Physics, Czech Academy of Sciences, Prague, Czech Republic

${ }^{35}$ Department of Physics and Institute for the Early Universe, Ewha Womans University, Seodaaemun-gu, Seoul, Korea

\section{Acknowledgements}

The Telescope Array experiment is supported by the Japan Society for the Promotion of Science(JSPS) through Grants-in-Aid for Priority Area 431, for Specially Promoted Research JP21000002, for Scientific Research (S) JP19104006, for Specially Promoted Research JP15H05693, for Scientific Research (S) JP15H05741 and JP19H05607, for Science Research (A) JP18H03705, for Young Scientists (A) JPH26707011, and for Fostering Joint International Research (B) JP19KK0074, by the joint research program of the Institute for Cosmic Ray Research (ICRR), The University of Tokyo; by the Pioneering Program of RIKEN for the Evolution of Matter in the Universe (r-EMU); by the U.S. National Science Foundation awards PHY-1404495, PHY-1404502, PHY-1607727, PHY-1712517, PHY-1806797 and PHY-2012934; by the National Research Foundation of Korea (2017K1A4A3015188, 2020R1A2C1008230, \& 2020R1A2C2102800) ; by the Ministry of Science and Higher Education of the Russian Federation under the contract 075-15-2020-778, RFBR grant 20-02-00625a (INR), IISN project No. 4.4501.18, and Belgian Science Policy under IUAP VII/37 (ULB). This work was partially supported by the grants of The joint research program of the Institute for Space-Earth Environmental Research, Nagoya University and Inter-University Research Program of the Institute for Cosmic Ray Research of University of Tokyo. The foundations of Dr. Ezekiel R. and Edna Wattis Dumke, Willard L. Eccles, and George S. and Dolores Doré Eccles all helped with generous donations. The State of Utah supported the project through its Economic Development Board, and the University of Utah through the Office of the Vice President for Research. The experimental site became available through the cooperation of the Utah School

\footnotetext{
${ }^{*}$ Deceased
} 
and Institutional Trust Lands Administration (SITLA), U.S. Bureau of Land Management (BLM), and the U.S. Air Force. We appreciate the assistance of the State of Utah and Fillmore offices of the BLM in crafting the Plan of Development for the site. Patrick A. Shea assisted the collaboration with valuable advice and supported the collaborationâ $\mathrm{tM}^{\mathrm{TM}} \mathrm{s}$ efforts. The people and the officials of Millard County, Utah have been a source of steadfast and warm support for our work which we greatly appreciate. We are indebted to the Millard County Road Department for their efforts to maintain and clear the roads which get us to our sites. We gratefully acknowledge the contribution from the technical staffs of our home institutions. An allocation of computer time from the Center for High Performance Computing at the University of Utah is gratefully acknowledged. 